\title{
PLASMA CELL LEUKEMIA- LIGHT CHAIN SECRETORY TYPE, WITH PRIMARY AMYLOIDOSIS
}

Dr Kiran. D. R, Dr Palaniswamy

1. Associate Professor, Department of General Medicine, Karuna Medical College, Vilayodi, Chittur, Palghat, Kerala.

2. Professor, Department of General Medicine, Karuna Medical College, Vilayodi, Chittur, Palghat, Kerala.

\section{CORRESPONDING AUTHOR:}

Dr. Kiran D R.,

Associate Professor,

Department of Medicine,

Karuna Medical College, Vilayodi,

Chittur, Palghat, Kerala.

Email id- kirandr_07@yahoo.co.in

\begin{abstract}
:
INTRODUCTION: Secondary PCL (Plasma Cell Leukemia), arising from multiple myeloma is aggressive and rare variant of multiple myeloma. It is known that AL (Amyloid Light Chain) type of Amyloidosis is primary amyloidosis occurring in multiple myeloma. Attempt to report this case of secondary PCL of light chain secretory type with primary amyloidosis. Emphasis given to this report is so as to highlight the helpful prognostic tools in this rare variety. CASE REPORT: 60 year old female presenting with Hepatosplenomegaly, severe anemia, bleeding manifestations and fever. She had pancytopenia with ESR of 170 . Peripheral smear showed plenty of plasma cells $>20 \%$ and bone marrow biopsy showed mature to immature plasma cells, plenty in number. Skull $\mathrm{X}$ ray showing multiple punched out lesions. Electrophoresis was normal, abdominal wall fat stained positive for Congo Red and Serum Free Light Chain Assay was positive. Diagnosed as PCL of light chain secretory type. CONCLUSION: PCL itself, high plasma cell labeling index, increased LDH, increased serum $\beta_{2}$ microglobulin,renal indices like increased serum creatinine, organomegaly, non secretory variety-all carry bad prognostic signs.
\end{abstract}

KEY WORDS: PCL, Primary Amyloidosis, Light chain, Punched out bone lesions

\section{INTRODUCTION:}

It is known that incidence of PCL is $<1 /$ million cases and $2 \%$ of multiple myeloma ${ }^{1}$. It is an aggressive and rare presentation of multiple myeloma ${ }^{2}$. It has features of acute leukemia and multiple myeloma.PCL usually arises from myeloma having IgD or IgE as M component. PCL, arising from pre existing multiple myeloma of light chain secretory type associated with AL (Amyloid Light chain) type of Amyloidosis is very rare. Since there are no large trials on treatment of patients with PCL, we here make an attempt report this case.

\section{CASE REPORT:}

A 60 years old female patient presented with history of fever and epistaxis of one month duration. On examination she was Febrile, having tachycardia and pallor. She had no generalized lymphadenopathy but had moderate Hepatosplenomegaly. She had sternal tenderness.

Journal of Evolution of Medical and Dental Sciences/Volume 1/Issue 3/July- Sept 2012 Page 106 


\section{INVESTIGATIONS:}

WBC count - 1, 22,000

$\mathrm{Hb}-2.8 \mathrm{mg} / \mathrm{dl}$

Platelet count $-80,000$

ESR- 170

Renal and Liver function tests are normal

Serum calcium: $12 \mathrm{mg} / \mathrm{dl}$

Serum uric acid: normal

Serum LDH: 310u/L

Serum $\beta_{2}$ microglobulin: $3.1 \mathrm{mg} / \mathrm{L}$

Serum Electrophoresis showed no M band

Serum Free Light Chain Assay: detected

Urine Bence Jones protein: Negative

Peripheral Smear Report: RBC showed hypochromia and anisocytosis. Neutrophils were decreased in number. A large number of plasma cells were seen .Immature and abnormal plasma cells were seen in plenty. A good number of binucleated plasma cells also seen. Immature cells exceeded 20\%. Platelets grossly decreased in number.

Bone Marrow Aspiration Report: Hypercellularmarrow.RBC precursors were reduced in number and were normopoietic. Granulocytic precursors were also reduced in number. Each field showed sheets of plasma cells. Many of the plasma cells were atypical. Some of them were rounded, some had nuclear abnormalities and some were binucleated. A large number of plasma blasts were also seen. Megakaryocytes were reduced in number.

Flow Cytometry of Bone Marrow Aspirate: Showed intense positivity for CD 38 and the presence of cytoplasmic kappa light chains.

Abdominal fat stained positive for Congo Red.

Skull X-ray showed multiple punched out lesions.

Our patient was diagnosed to have primary PCL - light chain type with Primary Amyloidosis, based on peripheral smear, bone marrow and flow cytometric analysis.

Treatment: Patient was given supportive treatment. Prednisolone and Melphelan were started. Patient expired in two months after diagnosis.

\section{DISCUSSION:}

Plasma cell leukemia is a lymphoproliferative disorder. It is a rare monoclonal neoplasm having $\mathrm{B}$ lymphocyte lineage. It is one of the aggressive human neoplasms accounting for $2-4 \%$ of all cases of plasma cell diseases ${ }^{3}$. Rarity of PCL can be accessed from the fact that at M.D. Anderson cancer centre, 27 patients with PCL were seen in 20 year period whereas at Policlinico-Sanmateo in Italy, 15 cases were seen in 15 years, both representing 2-5\% of total cases of multiple myeloma seen at these centers. Incidence of PCL is less than 1 case per million population ${ }^{1}$. Presentation may be primary, secondary - evolving from an existing case of multiple myeloma as part of the terminal phase of the disease or denova. Sixty to seventy percent cases are primary. PCL is of two types, Secretory and non-secretory. No M protein is detected in the non secretory type of PCL4.

WHO criteria to diagnose PCL are - Plasma cells constitute more than $20 \%$ of cells in the peripheral blood with an absolute plasma cell count of more than $2 \times 10^{9}$ / litre.

Lab findings: There is overlap between cells of multiple myeloma and Primary PCL. PCL plasma cells more frequently express the CD 20 antigen than those of multiple myeloma and they often

Journal of Evolution of Medical and Dental Sciences/Volume 1/Issue 3/July- Sept 2012 Page 107 
lack CD 56 antigen which is present on the majority of myeloma cells. CD 56 is important in anchoring plasma cells to bone marrow stroma and its expression is associated with poor prognosis. CD 28 is more frequently expressed on malignant plasma cells in secondary than in primary PCL. Acquisition of CD 28 is associated with increased proliferative rate and disease progression. PCL is derived from terminally differentiated $B$ cells and the malignant cells stain positive for mature B cell markers (CD38 and PCA1, Prostate Cancer Antigen 1) . $^{5}$

In $80 \%$ of PCL patients, using FISH (Flourescent In Situ Hybridisation) technique- losses in long arm of chromosome $13(13$ Q) and deletion of one of the homologous chromosome 13 (Monosomy 13). In $80 \%$ of cases, loses on chromosome 16 also occur. Overexpression of PRAD1/cyclinD1 (Parathyroid associated Neoplasia gene) also present (help in controlling cell cycle). PCL is more frequent in light chain (Bence Jones's protein) or IgD myeloma and less in IgA or IgG myeloma. Hypercalcaemia and increased LDH are known bad prognostic signs of disease ${ }^{6}$. PCL is an extremely aggressive disease with no standard treatment regimen so far due to the rarity of the disease. Median survival is 2-8 months, prognosis is very poor. Regimens includes MP, (Melphelan, Prednisolone) VAD (Vincristine, Doxorubicin, Dexamethasone) TD (Thalidomide, Dexamethasone) VBAP (Vincristine, Carmustine, Adriamycin, Prednisolone) ${ }^{7}$. Case reports regarding long time survival after autologous bone marrow transplantation or stem cell transplantation also exists but again there is no long term prospective data on a large number of patients. We presented this case for its rarity.

\title{
CONCLUSION:
}

PCL itself has aggressive course, short survival time, high plasma cell labeling index, expression of CD 28, Cyclin, PRAD 1 indicate high proliferation. Lytic bone lesion is more common in multiple myeloma than PCL. Biochemical parameters like increased serum creatinine, LDH, Serum $\beta_{2}$ microglobulin and calcium have poor prognosis. Amyloidosis associated multiple myeloma indicate tumor burden. Non secretory variety has got bad prognosis. So above mentioned prognostic tools may help to judge the severity of the tumour burden in a PCL case and act accordingly

\author{
ABBREVIATIONS: \\ PCL: Plasma Cell Leukemia \\ LDH: Lactate Dehydrogenase \\ CD: Cluster Differentiating \\ AL: Amyloid Light
}

\section{ACKNOWLEDGEMENT:}

I take this opportunity to extend my sincere thanks and indebtedness to all those persons and dignitaries who helped me to complete this work.

It gives me pleasure to express my sense of gratitude to my Professor Dr Palaniswamy, for their guidance, encouragement and constant source of inspiration during case management. Above all I thank the Almighty for the successful completion of this work. 


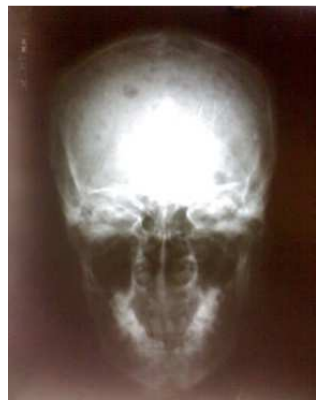

Skull X Ray PA view- Showing punched out lesions

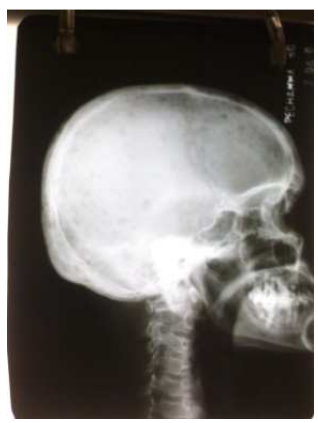

Skull X Ray Lateral view- Showing punched out lesions

\section{REFERENCES:}

1. Kosma MA, Gale RP. Plasma Cell Leukemia.SeminHaematology 1987.24; 202-8

2. DimopoulasMA,PalumboA,DelasalleKB,AlexanianR.Primary Plasma Cell leikamia.Br Journal Hematol 1994;88;754-9.

3. Chan SM, George T, Cherry AM,Medeiras BC(2009) Complete remission of primary PCL Bortezomito, Doxorubicin and Dexamethasone; a case report; cases 12: 1186/17571626-2.

4. ShindoT,Yumoto Y, Yoshida M, Okuda T; Non Secretory Primary Plasma Cell Leukemia successfully treated with VAD and MP therapy.RhinshoKetsueki; 2002;43; 107-11

5. Badhe BA, Basu D, Toe P Ch,Dutta TK, Ghotekar LH; Plasma Cell Leukemia; a case report. Indian Journal Pathology Microbiology 2003; 46; 484-7.

6. Voutsadakis IA; Plasma Cell Leukemia. Hama 2000; 3:82-9.

7. Suzuki M, Kawauchi K, Sugiyama H, Yasuyama M, Watanabe H. Primary Plasma Cell Leukemia; a case report of successful responder to a combination of Vincristine, Doxorubicin and Dexamethasone. ActaHematol 1989; 82:95-7. 